\title{
A Comparison of Two Formulations to Blend Finite Element and Mesh-Free Methods
}

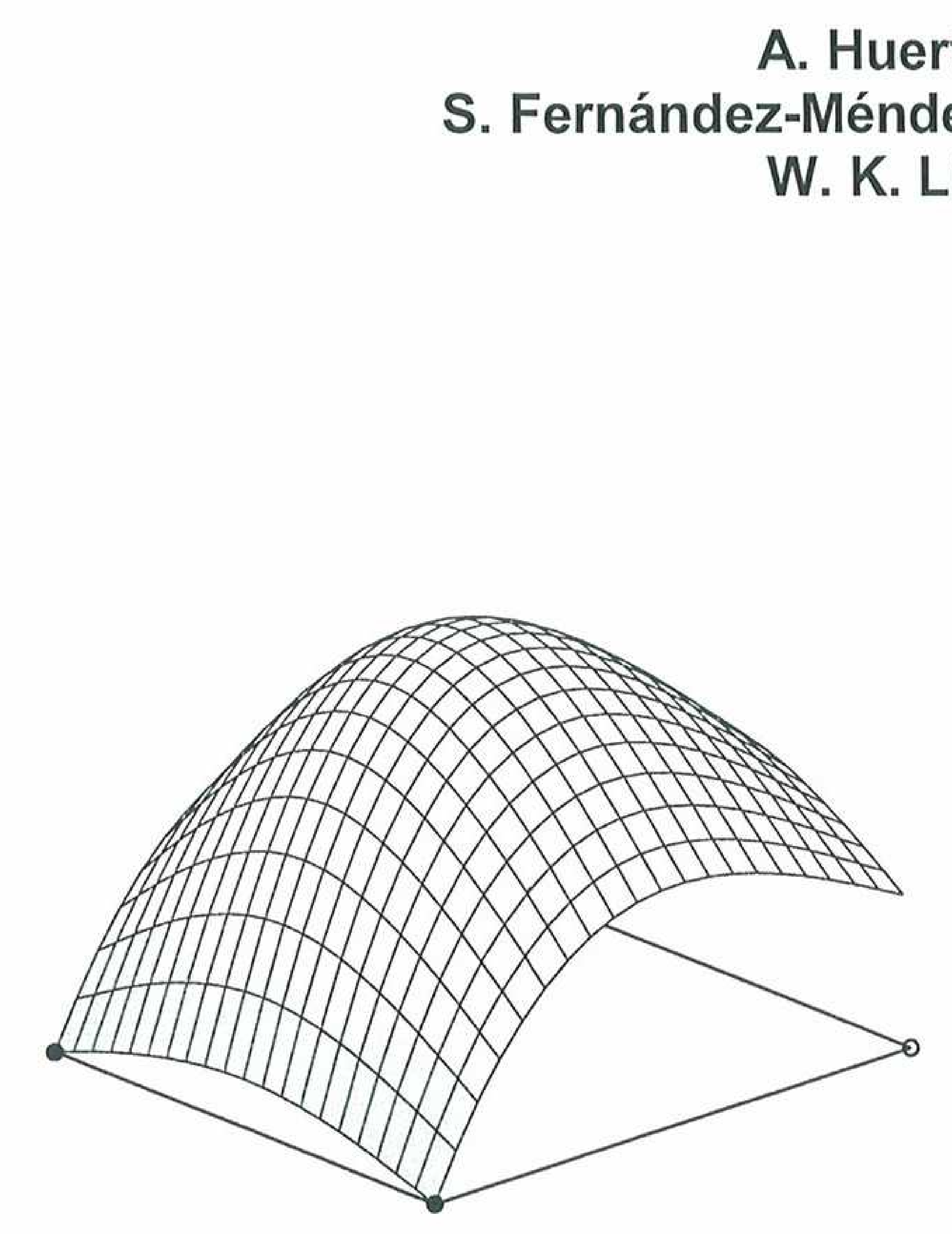




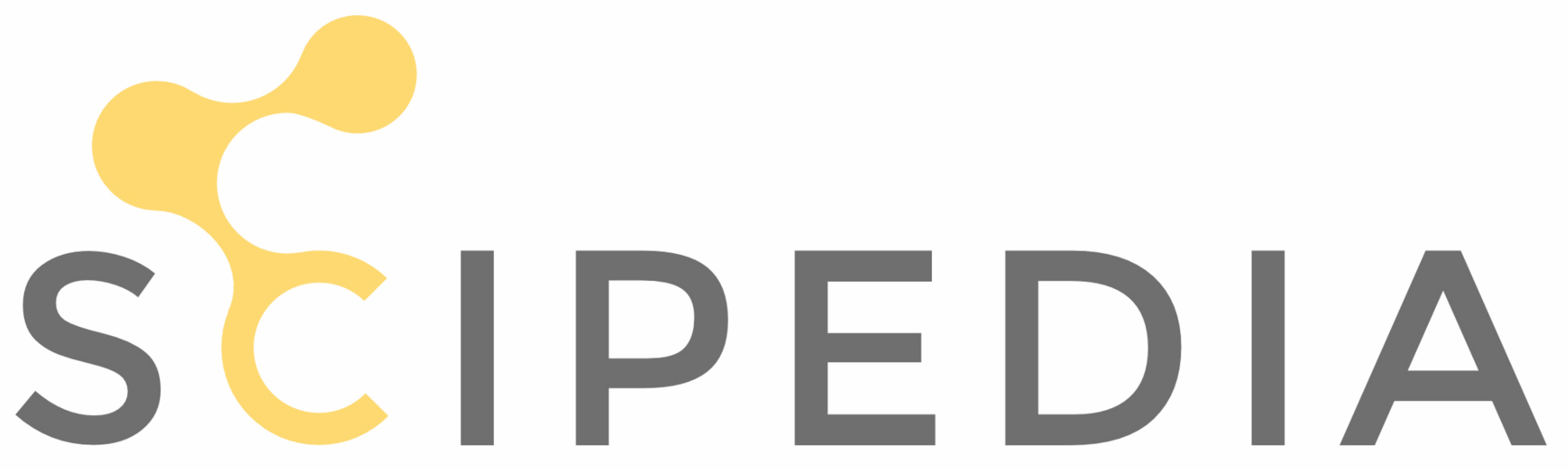

Register for free at https//www.scipedia.com to download the version without the watermark 


\section{A Comparison of Two Formulations to Blend Finite Element and Mesh-Free Methods}

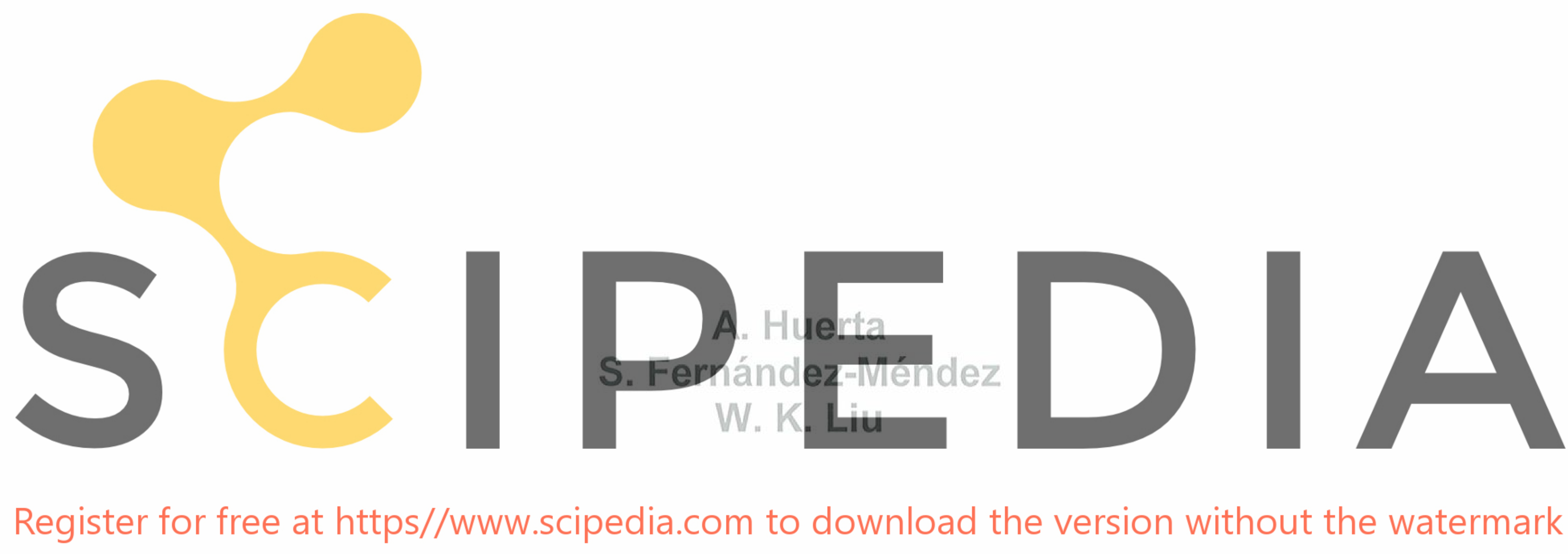

Publication CIMNE Nº-235, July 2003 


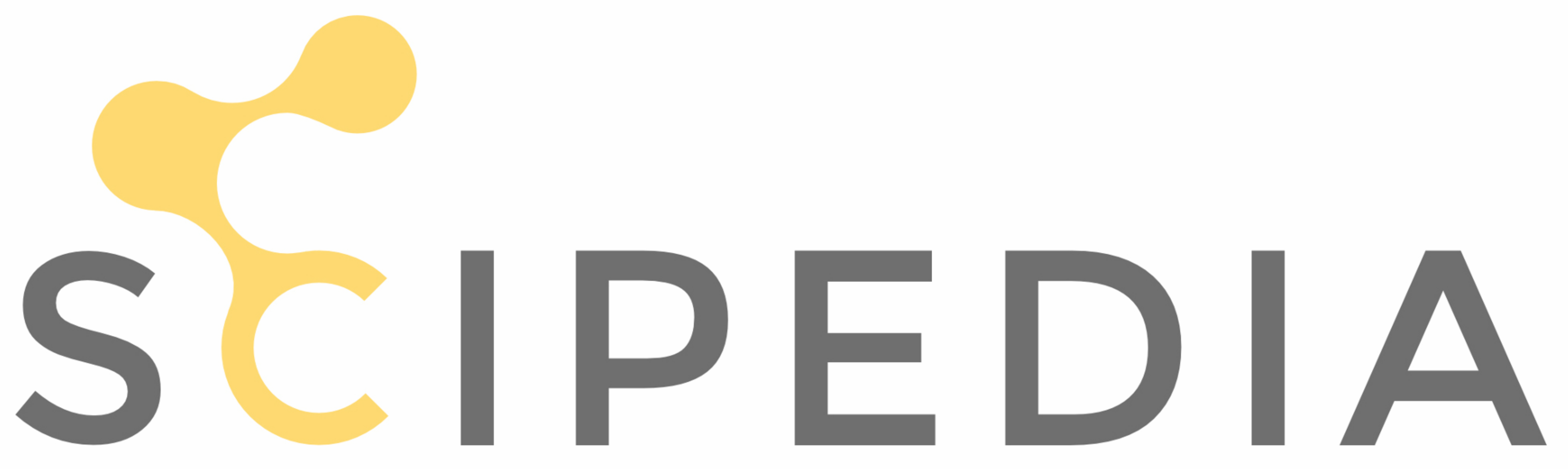

Register for free at https//www.scipedia.com to download the version without the watermark 


\title{
A comparison of two formulations to blend finite elements and mesh-free methods
}

\author{
Antonio HUERTA ${ }^{\text {a,*,1, Sonia FERNÁNDEZ-MÉNDEZ }}{ }^{\mathrm{a}, 1}$ and \\ Wing Kam LIU ${ }^{\text {b,2 }}$
}

${ }^{a}$ Laboratori de Càlcul Numèric, Universitat Politècnica de Catalunya, Jordi Girona 1, E-08034 Barcelona, Spain.

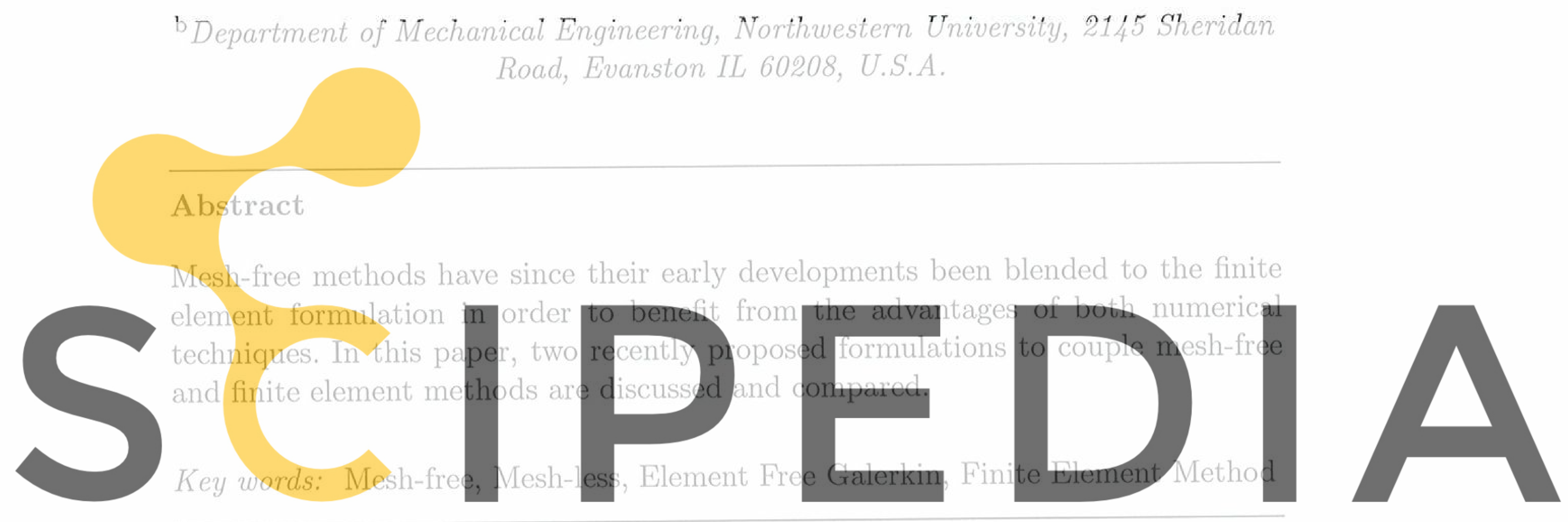

Register for free at https//www.scipedia.com to download the version without the watermark

\section{Introduction}

Seminal work on coupling finite elements and mesh-free methods can be found in [1]. The objective is always to use the advantages of each method. They show how to couple finite elements near the Dirichlet boundaries and element-free Galerkin in the interior of the computational domain. This simplifies considerably the prescription of essential boundary conditions. They do a mixed interpolation in the transition region: area where both finite elements and particles have an influence. This mixed interpolation requires the substitution

* Correspondence to: Antonio Huerta, Departament de Matemàtica Aplicada III, E.T.S. de Ingenieros de Caminos, Canales y Puertos, Universitat Politècnica de Catalunya, Jordi Girona 1, E-08034 Barcelona, SPAIN.

URL: www-lacan. upc.es (Antonio HUERTA).

1 Sponsored by Ministerio de Ciencia y Tecnología (grants: DPI2001-2204 and REN2001-0925-C03-01) and the Generalitat de Catalunya (grant: 2001SGR00257)

2 Sponsored by NSF 
of finite element nodes by particles and the definition of ramp functions. Thus the region for transition is of the size of one finite element and the interpolation in linear. With the same objectives in [2] the finite element domain and the mesh-free region are coupled with Lagrange multipliers.

Here two recently proposed formulations to couple mesh-free and finite element methods are discussed and compared. They generalize the ideas of [1] for any order of interpolation, suppress the ramp functions, and do not require the substitution of nodes by particles. That is, as many particles as needed can be added where they are needed independently of the adjacent finite element mesh. This is done in a hierarchical manner and enforcing uniform consistency for the blended interpolation. In [3] a coupled hierarchical approximation method is proposed, see also [4-6]. Liu and co-workers have also proposed a hierarchical enrichment for bridging scales [7-10]. The advantages and disadvantages of both formulations are presented and discussed; moreover, numerical examples illustrating this issues are presented.

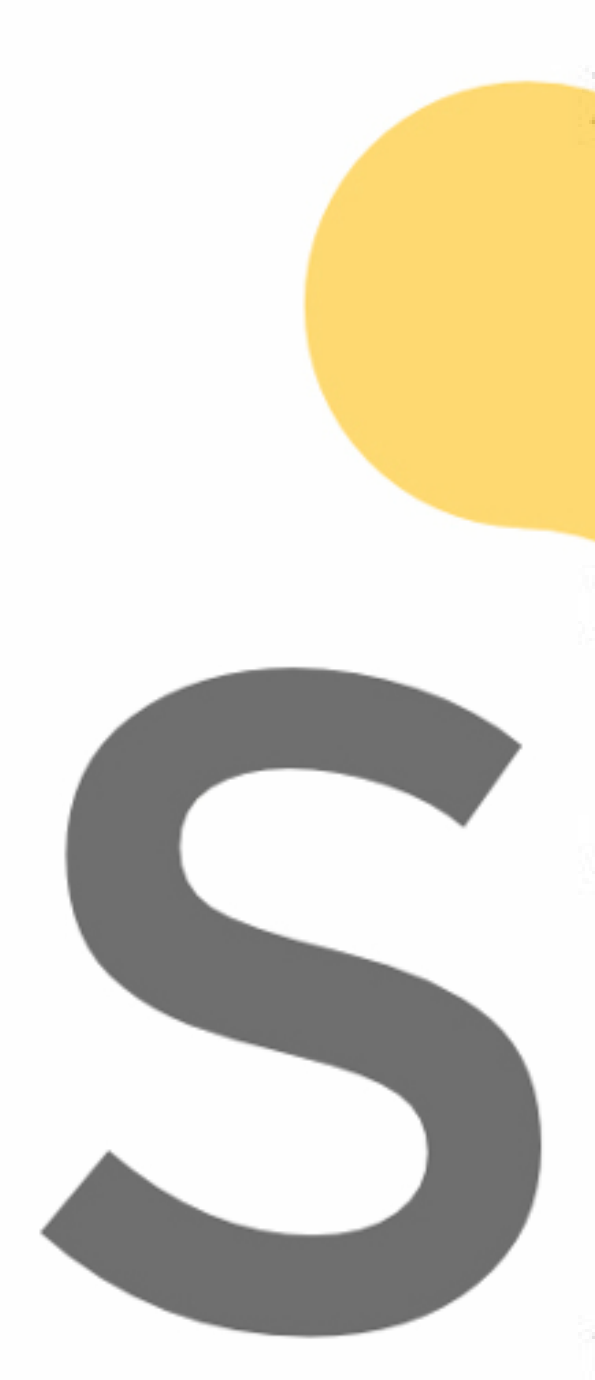

2 Two consistent hierarchical formulations

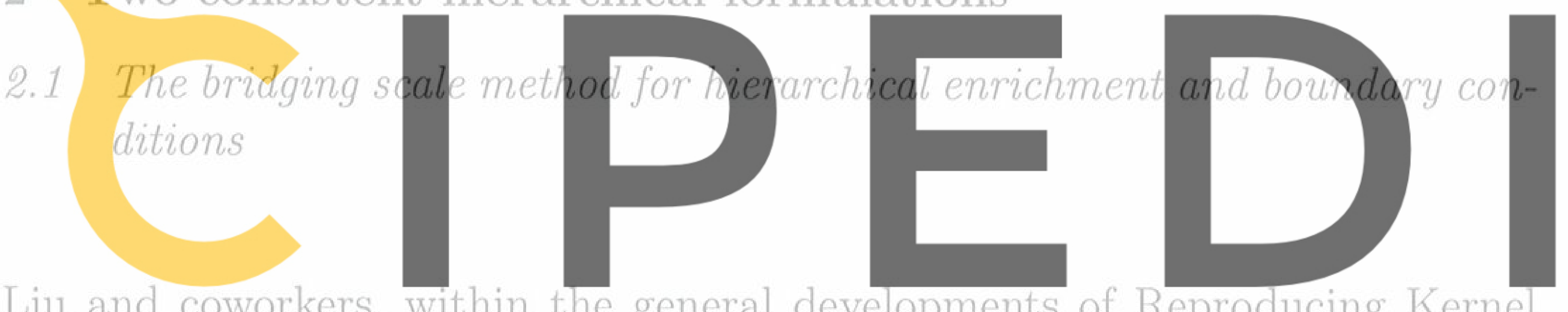

Liu and coworkers, within the general developments of Reproducing Kernel

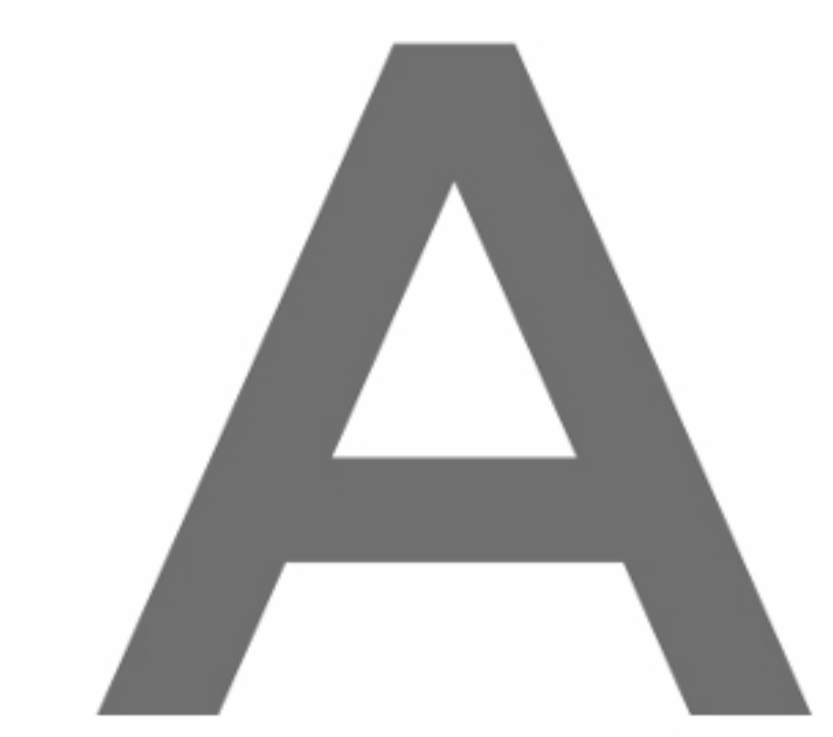

Register for free at https//www.scipedia.com to download the version without the watermark lows a mesh-liee approach and at the same time enriches a standard finite

element approximation [7-10]. The basic concept is the hierarchical decomposition of a function $u$ based on some projector $\pi^{h}$. In general $\pi^{h}$ is a projection operator onto any approximation space, but usually a projection onto a finite element space is employed $[7,8]$. That is, the span of some finite element shape functions characterized by an element mesh size $h$. The rationale is to enrich a non-complete finite element base (viz., a finite element base whose supports do not cover the whole domain) with a mesh-free interpolation. In this approach particles must be added in the whole domain in order to recover the completeness of the interpolation. That is, the distribution of particles over the whole domain is such that the necessary conditions for solvability are met at every point of the domain $[13,3,5]$. Figure 1 presents an example. It shows a spatial domain where finite element nodes are considered only along the Dirichlet boundary. Those are the active nodes for the functional interpolation. Other non-active nodes are considered to define the support of the shape functions (thus only associated to the geometrical interpolation), see Figure 1.

Thus, designating as $\mathcal{B}$ the set of indexes of the active nodes, $\left\{x_{j}\right\}_{j \in \mathcal{B}}$, the 


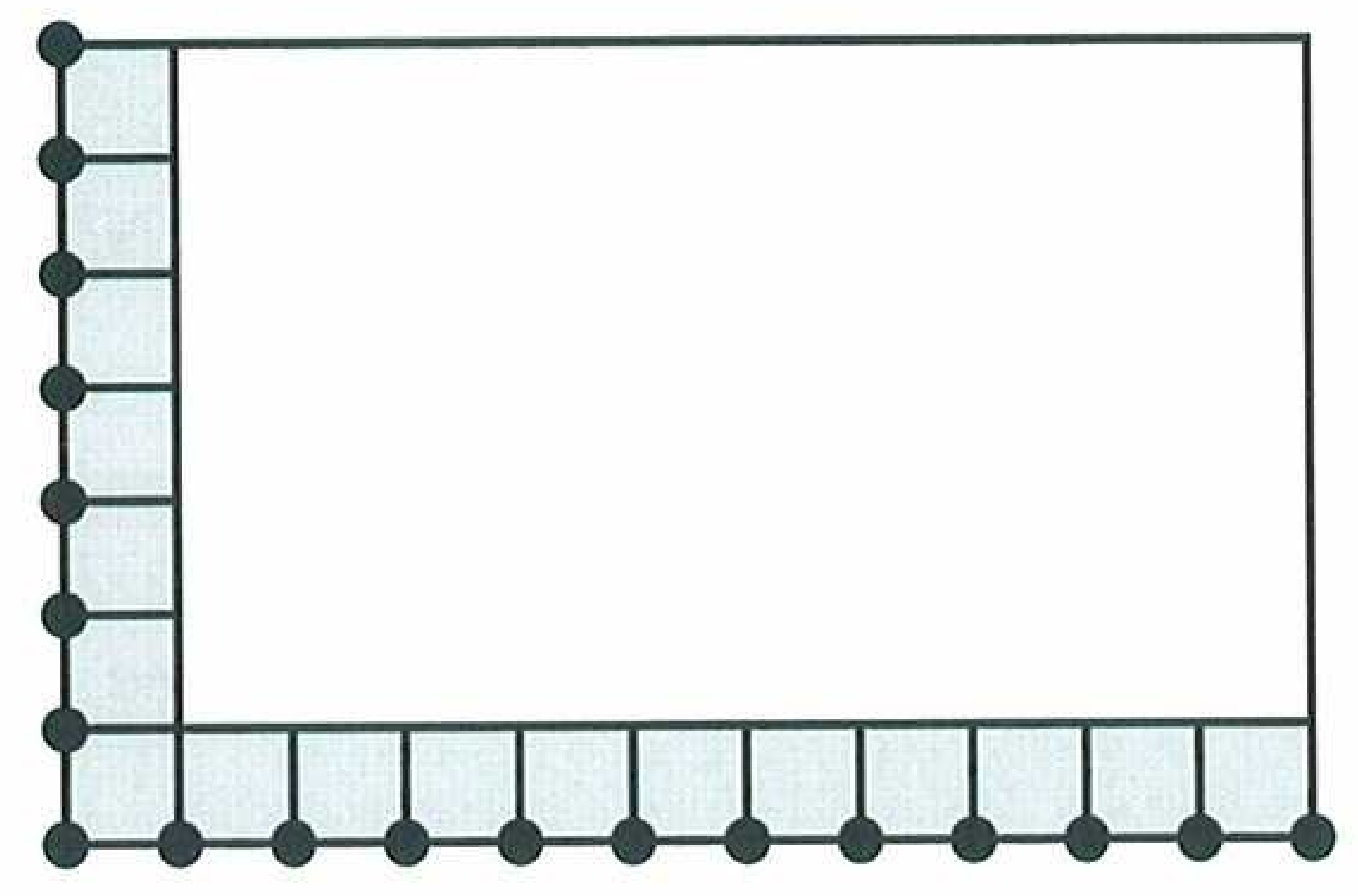

Fig. 1. Finite element nodes $(\bullet)$ and support of the incomplete base of finite element shape functions (in gray).

projection onto the incomplete finite element base is

$$
\pi^{h} u(x)=\sum_{j \in \mathcal{B}} N_{j}^{h}(x) a_{j}
$$

where $a_{j}$ are the coefficients, which must coincide with $u\left(x_{j}\right)$, and $N_{j}^{h}(x)$ are the finite element shape functions.
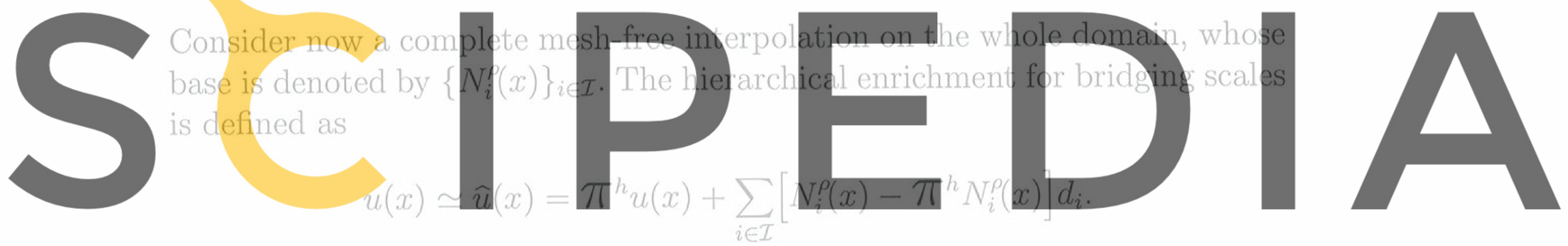

Register for free at https//www:scipedia,com to download the version without the watermark

$$
u(x) \simeq \widehat{u}(x)=\sum_{j \in \mathcal{B}} N_{j}^{h}(x) a_{j}+\sum_{i \in \mathcal{I}} N_{i}^{P}(x) d_{i}-\sum_{i \in \mathcal{I}} \sum_{j \in \mathcal{B}} N_{j}^{h}(x) N_{i}^{P}\left(x_{j}\right) d_{i}
$$

or as

$$
u(x) \simeq \widehat{u}(x)=\sum_{j \in \mathcal{B}} N_{j}^{h}(x) a_{j}+\sum_{i \in \mathcal{I}} \widehat{N}_{i}^{\rho}(x) d_{i},
$$

where $\widehat{N}_{i}^{\rho}=N_{i}^{\rho}-\pi^{h} N_{i}^{\rho}$ are the hierarchical modified mesh-free shape functions.

Remark 1 This interpolation is hierarchical because

$$
\widehat{N}_{i}^{\rho}\left(x_{j}\right)=N_{i}^{\rho}\left(x_{j}\right)-\left[\pi^{h} N_{i}^{\rho}\right]\left(x_{j}\right)=0 \quad \text { for } j \in \mathcal{B} .
$$

This result is obvious from the last two terms on the r.h.s. of (1) and the delta property of the finite element shape functions, $N_{j}^{h}\left(x_{i}\right)=\delta_{i j}$ for $i$ and $j$ in $\mathcal{B}$. Thus the enrichment does not affect the nodal values. That is, the approximation values of $\widehat{u}$ at the active nodes are not modified by the meshfree interpolation. 
Remark 2 The approximation (2) can also be written as

$$
\widehat{u}(x)=\sum_{j \in \mathcal{B}} N_{j}^{h}(x) c_{j}+\sum_{i \in \mathcal{I}} N_{i}^{\rho}(x) d_{i} \quad \text { where } c_{j}=a_{j}-\sum_{i \in \mathcal{I}} N_{i}^{\rho}\left(x_{j}\right) d_{i} .
$$

Thus, the approximation space in this hierarchical enrichment, which from expression (2) appears to be $\operatorname{span}\left\{N_{j}^{h}, j \in \mathcal{B}\right\} \oplus \operatorname{span}\left\{\widehat{N}_{i}^{\rho}, i \in \mathcal{I}\right\}$, is nothing else but the direct sum of the finite element and the mesh-free interpolation, namely

$$
\widehat{\mathcal{S}}:=\operatorname{span}\left\{N_{j}^{h}, j \in \mathcal{B}\right\} \oplus \operatorname{span}\left\{N_{i}^{\rho}, i \in \mathcal{I}\right\} .
$$

Note that the finite element base is not complete almost everywhere. Only along the boundary defined by the active nodes, see Figure 1, the finite element base induces the desired consistency. Thus consistency is guaranteed by the meshfree approximation both in the region where particles are alone, as usual, and also in the region where the finite element base is incomplete, the gray area in Figure 1.

Remark 3 The shape functions $\left\{N_{j}^{h}\right\}_{j \in \mathcal{B}}$ and $\left\{\widehat{N}_{i}^{\rho}\right\}_{i \in \mathcal{I}}$ are linearly independent as long as the finite element base is not a complete base. If active nodes are only distributed on the boundary this is verified automatically. In fact, if

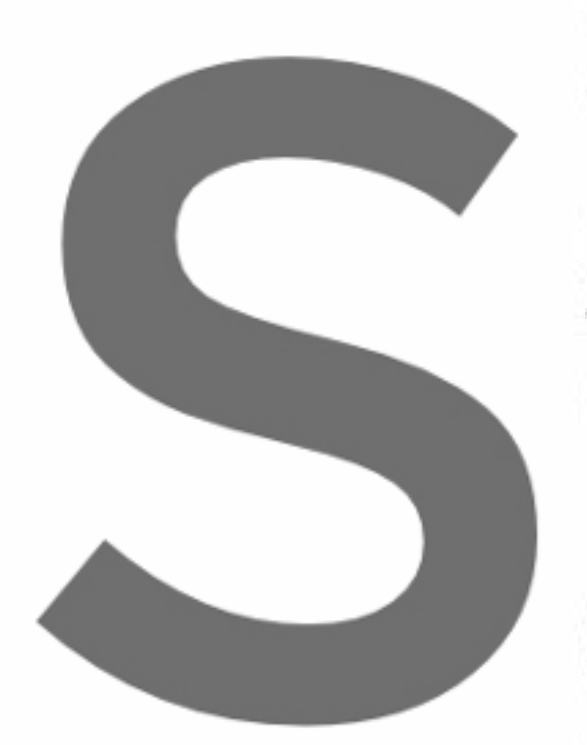
$\sum_{j \in \mathcal{B}} N_{j}^{h} \neq 1$ the base is
active.
Since this enrtchment is not
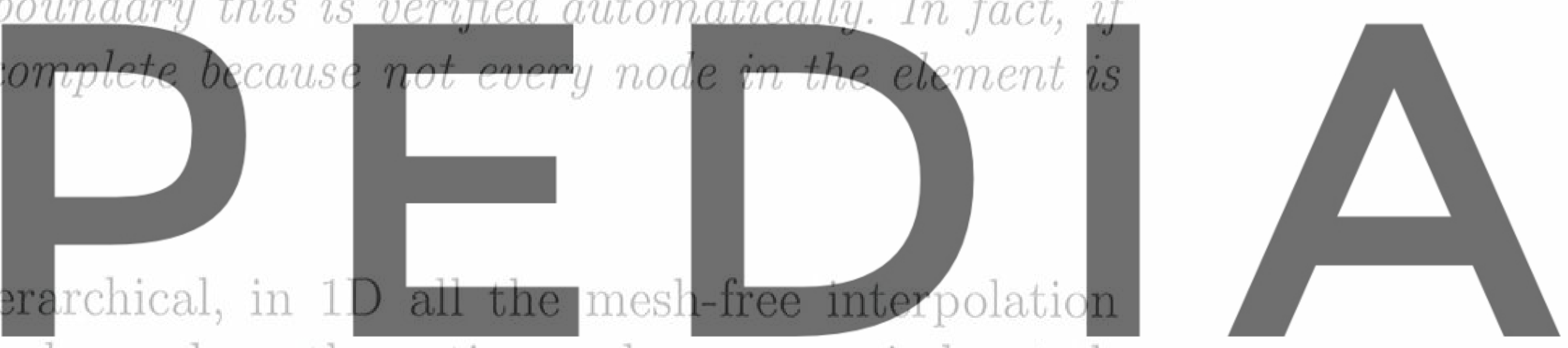

functions cancel on the boundary where the active node, say $x_{D}$, is located,

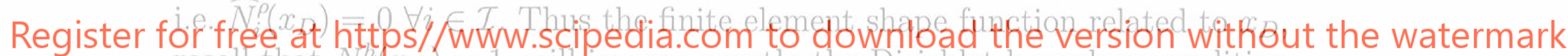

recall that $N_{D}^{\prime}\left(x_{D}\right)=1$, will impose exactly the Dirichlet boundary condition as desired.

However, this property is not generalizable to higher dimensions. Although the mesh-free shape functions vanish at the finite element nodes, they do not vanish along the element boundaries (edges in 2D and faces in 3D). Figure 2 shows an example of such a case. The shape function of a particle positioned in the center of a 2D element with two active nodes is hierarchical, i.e. goes to zero at the active nodes, but it does not cancel along the edge between those nodes. This fact induces important errors in the implementation of Dirichlet boundary conditions as already noticed in [8, sec. 5.2.1]. Convergence of this approach is still guarantee but at a lower rate than expected [9].

\subsection{Continuous blending method}

Another alternative for a hierarchical enrichment is the technique proposed in [3], which has been generalized in [14] to get a nodal interpolation property, 


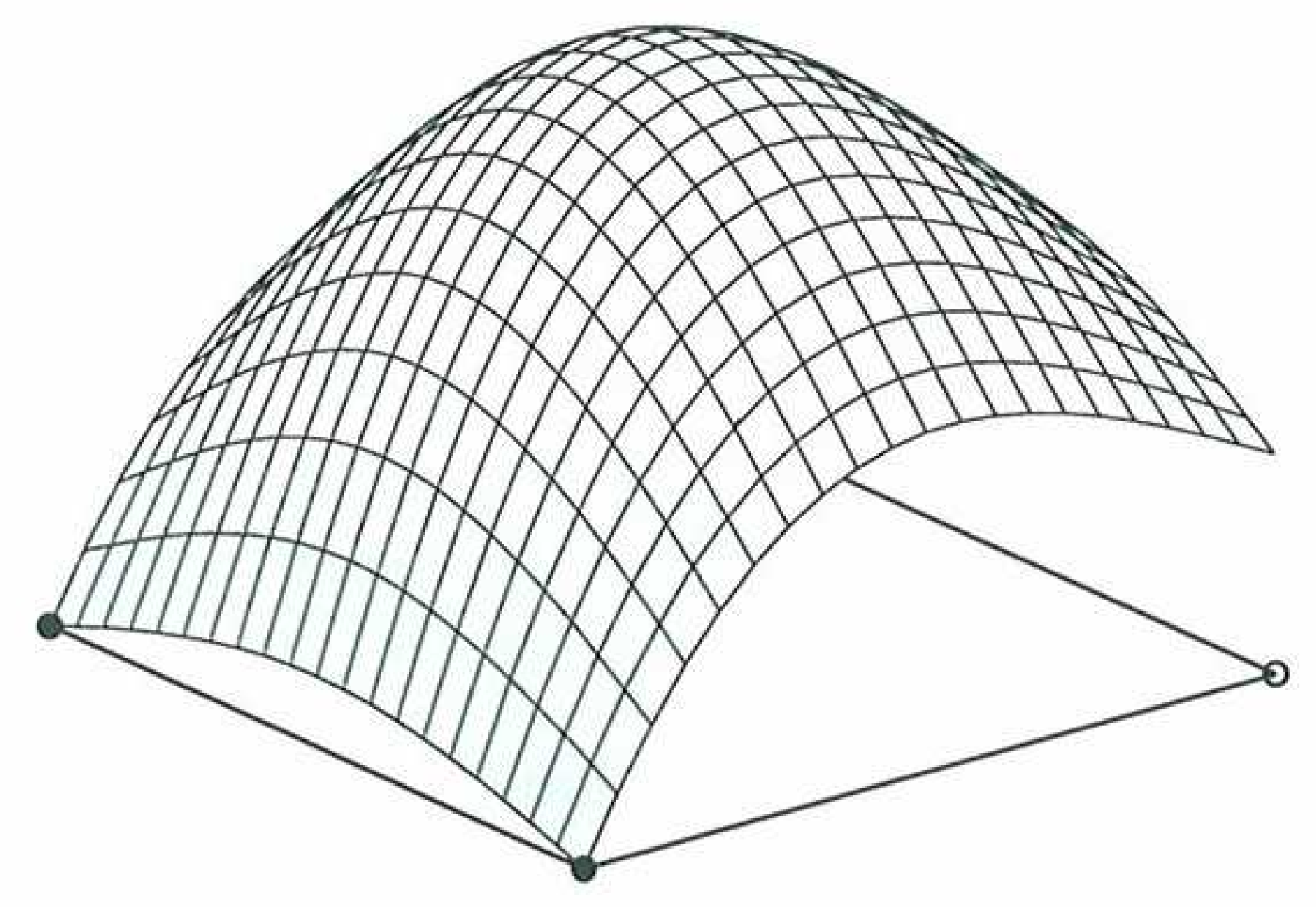

Fig. 2. Bridging scale mesh-free shape function over an element with two active nodes.

and whose convergence has been analyzed in [4-6]. This approach follows a different perspective: instead of doing a projection onto the finite element space, the computation of the mesh-free shape functions is adapted to account for the complete and incomplete finite element base.

In the context of the EFG method, given a set of particles $\left\{x_{i}\right\}_{i \in \mathcal{I}}$ in $\Omega$, the mesh-free shape functions can be written as
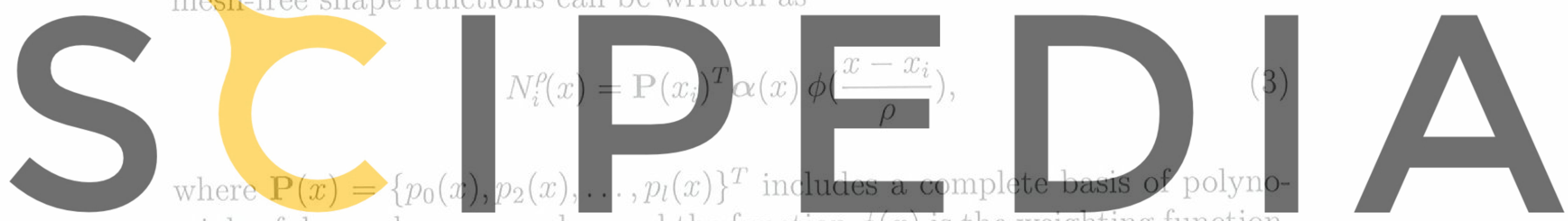

mials of degree less or equal $m$ and the function $\phi(x)$ is the weighting function

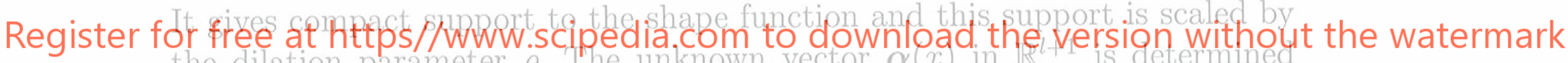

the dilation parameter $\rho$. Lhe unknown vector $\alpha(x)$ the

imposing the so-called reproducibility or consistency condition. In fact, it is

equivalent to a Moving Least Squares development [15]. This reproducibility condition imposes that the mesh-free approximation is exact for all the polynomials in $\mathrm{P}$, i.e. $\mathrm{P}(x)=\sum_{i} \mathrm{P}\left(x_{i}\right) N_{i}^{\rho}(x)$.

Following this idea Huerta and coworkers propose a combined approximation with finite elements and nodes,

$$
u(x) \simeq \widetilde{u}(x)=\sum_{j \in \mathcal{J}} N_{j}^{h}(x) u_{j}^{h}+\sum_{i \in \mathcal{I}} \widetilde{N}_{i}^{\rho}(x) u_{i}^{\rho}=\pi^{h} u+\sum_{i \in \mathcal{I}} \widetilde{N}_{i}^{\rho}(x) u_{i}^{\rho}
$$

where the finite element shape functions $\left\{N_{j}^{h}\right\}_{j \in \mathcal{J}}$ are as usual, and the meshfree shape functions $\left\{\widetilde{N}_{i}^{\rho}\right\}_{i \in \mathcal{I}}$ take care of the consistency of the the approximation. In Figure 3 this is clearly shown, the mesh-free shape functions adapt their shape to recover the linear interpolation. Note that the coefficients of the approximation are denoted by $u^{h}$ and $u^{\rho}$ to clearly identify those related to the finite element and to the mesh-free shape functions, respectively. The mesh-free shape functions are defined as in standard EFG, 


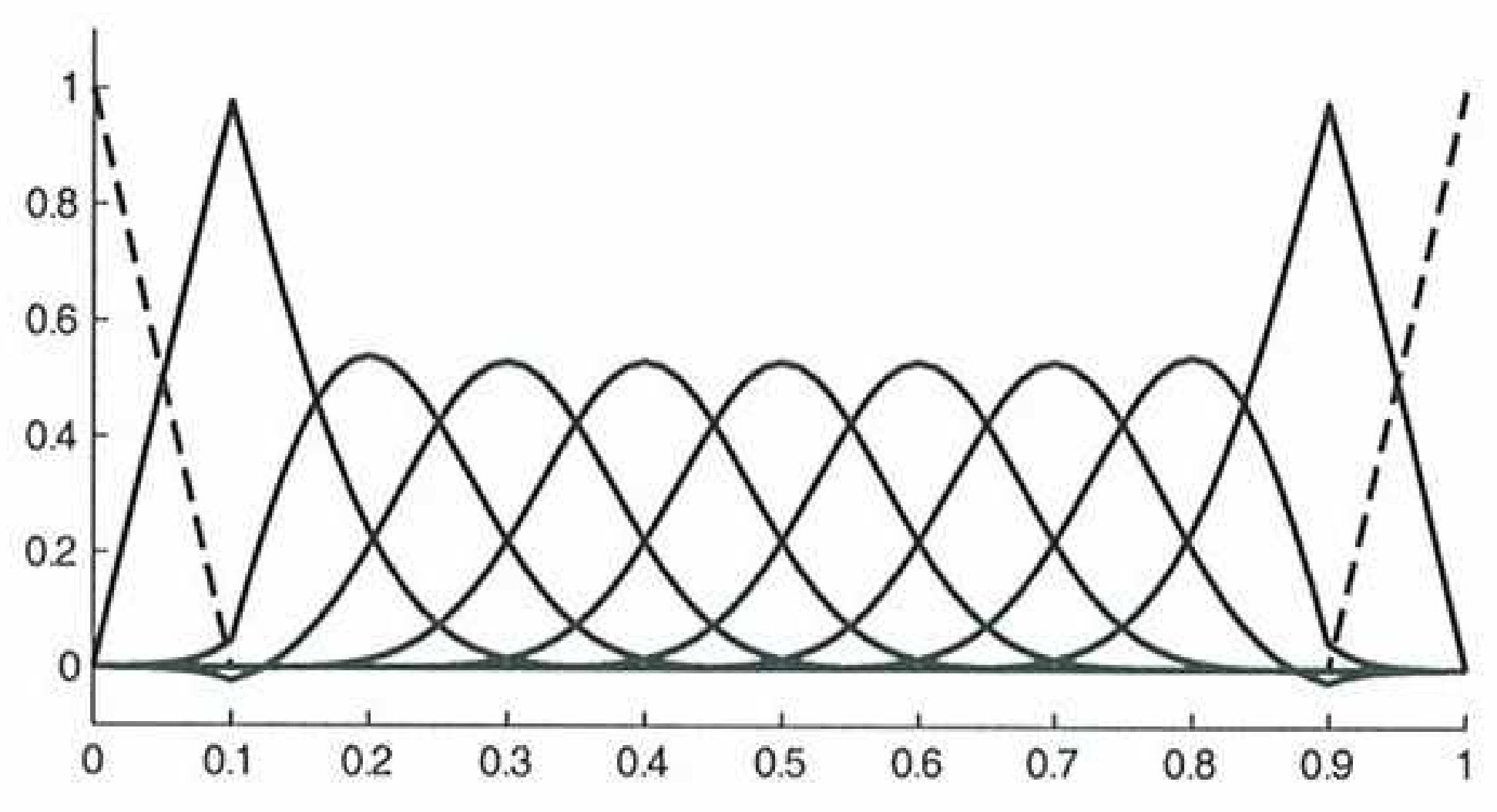

Fig. 3. Continuous blending method: the mesh-free shape functions recover the linear consistency.

$$
\widetilde{N}_{i}^{\rho}(x)=\mathbf{P}\left(x_{i}\right)^{T} \widetilde{\boldsymbol{\alpha}}(x) \phi\left(\frac{x-x_{i}}{\rho}\right),
$$

but the unknown vector $\widetilde{\alpha}$ is determined imposing the reproducibility condition associated to the combined approximation (4), that is

$$
\mathbf{P}(x)=\pi^{h} \mathbf{P}(x)+\sum_{i \in \mathcal{I}} \widetilde{N}_{i}^{\rho}(x) \mathbf{P}\left(x_{i}\right) .
$$

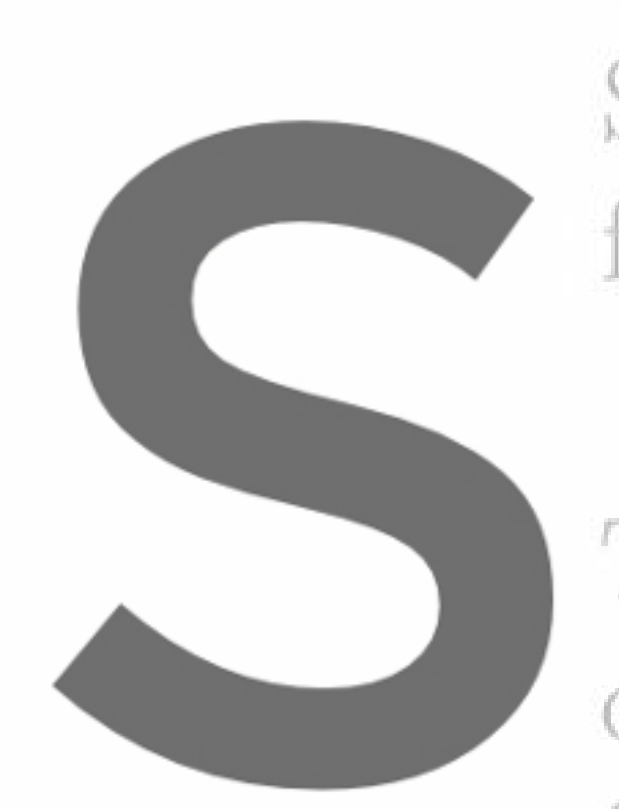

Sulbstitution of (5) for detaits,

The only one differen of the previous system
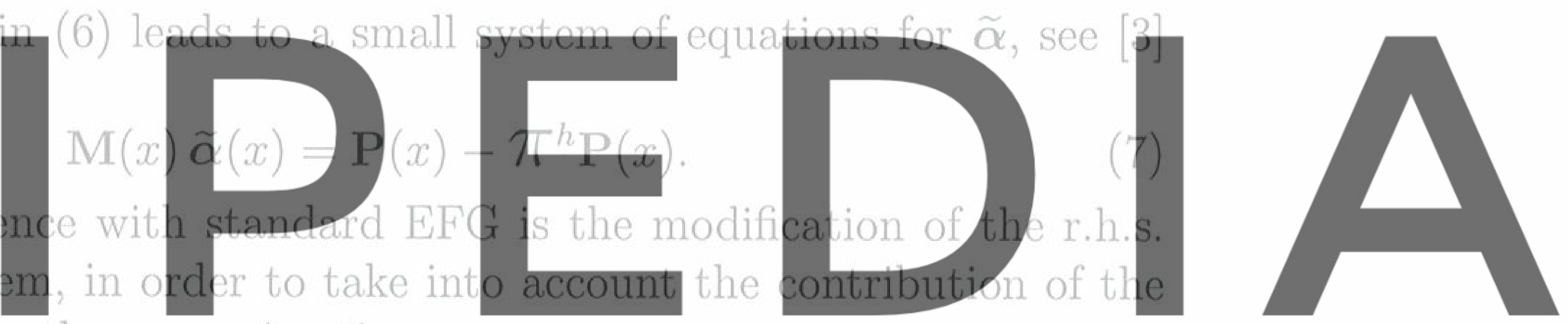

finite element base in the approximation.

Register for free at https//www.scipedia.com to download the version without the watermark functions must exactly reproduce $\mathrm{P}(x)-\pi^{h} \mathrm{P}(x)$, that is particles take care of the error due to the projection onto the finite element base.

In fact, thanks to the linearity of the system of equations defined by (7), $\widetilde{\alpha}$ is easily identified as

$$
\widetilde{\alpha}(x)=\alpha(x)-[\mathrm{M}(x)]^{-1} \pi^{h} \mathrm{P}(x),
$$

where $\boldsymbol{\alpha}(x)=[\mathbf{M}(x)]^{-1} \mathbf{P}(x)$ are the coefficients obtained in a pure mesh-free strategy. Likewise, the shape functions, see (5), can be identified as composed of two parts: the standard mesh-free one and the one accounting for the finite element contribution, namely $\widetilde{N}_{i}^{\rho}(x)=N_{i}^{\rho}(x)-\widetilde{\widetilde{N}}_{i}^{\rho}(x)$ with $N_{i}^{\rho}(x)$ defined in (3) and

$$
\begin{aligned}
\widetilde{\widetilde{N}}_{i}^{\rho}(x) & =\mathbf{P}\left(x_{i}\right)^{T}[\mathbf{M}(x)]^{-1} \pi^{h} \mathbf{P}(x) \phi\left(\frac{x-x_{i}}{\rho}\right) \\
& =\sum_{j \in \mathcal{J}} N_{j}^{h}(x) \mathbf{P}\left(x_{i}\right)^{T}[\mathbf{M}(x)]^{-1} \mathbf{P}\left(x_{j}\right) \phi\left(\frac{x-x_{i}}{\rho}\right)=\sum_{j \in \mathcal{J}} N_{j}^{h}(x) \overline{\bar{N}}_{i}^{\rho}\left(x, x_{j}\right) .
\end{aligned}
$$




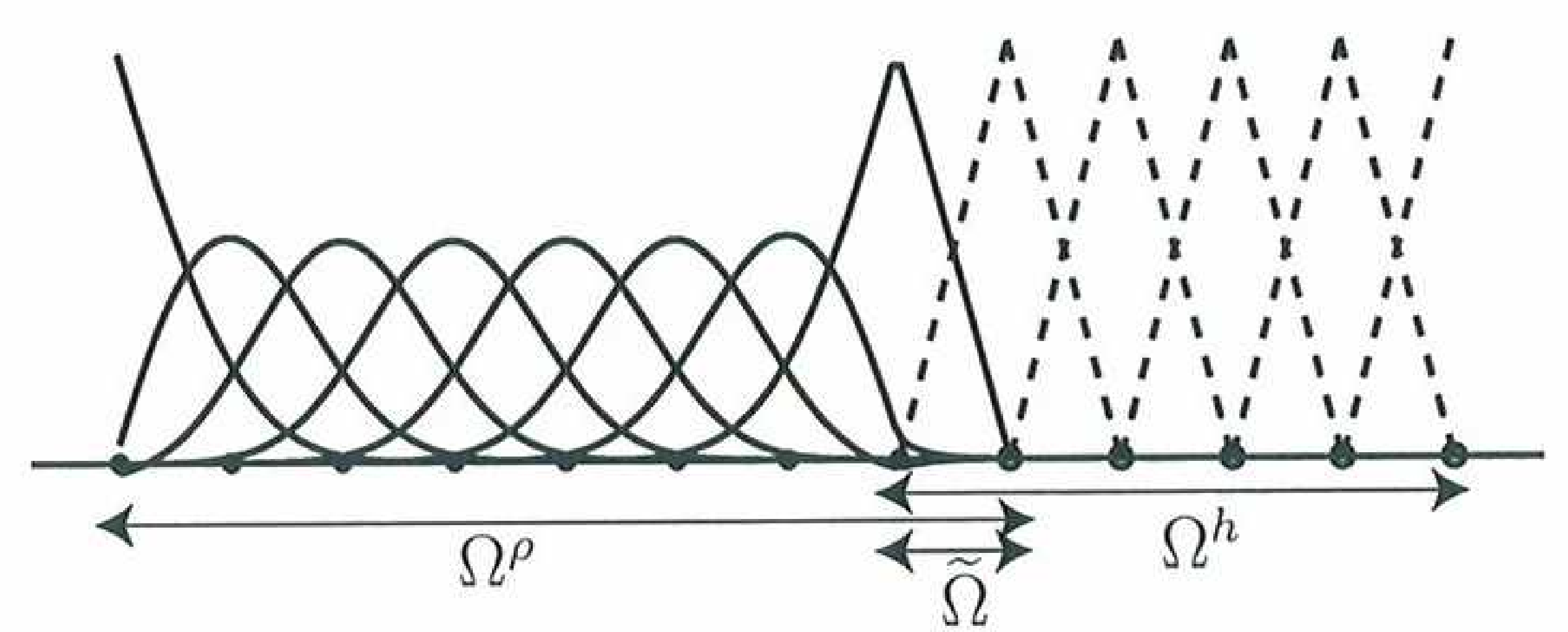

Fig. 4. Shape functions of the continuous blending method with coupled finite element (solid line) and mesh-free (dashed line) interpolation.

Thus after substitution in (4) another equivalent expression of the continuous blending method can be obtained

$$
\begin{aligned}
u(x) \simeq \widetilde{u}(x) & =\sum_{j \in \mathcal{J}} N_{j}^{h}(x) u_{j}^{h}+\sum_{i \in \mathcal{I}} N_{i}^{\rho}(x) u_{i}^{\rho}+\sum_{i \in \mathcal{I}} \widetilde{\widetilde{N}}_{i}^{\rho}(x) u_{i}^{\rho} \\
& =\sum_{j \in \mathcal{J}} N_{j}^{h}(x) u_{j}^{h}+\sum_{i \in \mathcal{I}} N_{i}^{\rho}(x) u_{i}^{\rho}+\sum_{i \in \mathcal{I}} \sum_{j \in \mathcal{J}} N_{j}^{h}(x) \overline{\bar{N}}_{i}^{\rho}\left(x, x_{j}\right) u_{i}^{\rho},
\end{aligned}
$$

which has a similar structure as (1).

Remark 5 This interpolation is hierarchical because at any finite element node $x_{k}, k \in \mathcal{J}$,

$$
\sum_{i \in \mathcal{I}} \sum_{j \in \mathcal{J}} N_{j}^{h}\left(x_{k}\right) \overline{\bar{N}}_{i}^{\rho}\left(x_{k}, x_{j}\right) u_{i}^{\rho}=\sum_{i \in \mathcal{I}} \sum_{j \in \mathcal{J}} \delta_{j k} \overline{\bar{N}}_{i}^{\rho}\left(x_{k}, x_{j}\right) u_{i}^{\rho}=\sum_{i \in \mathcal{I}} \overline{\bar{N}}_{i}^{\rho}\left(x_{k}, x_{k}\right) u_{i}^{\rho},
$$

and the function $\overline{\bar{N}}_{i}^{\rho}\left(x, x_{k}\right)$ is such that $\overline{\bar{N}}_{i}^{\rho}\left(x_{k}, x_{k}\right)=N_{i}^{\rho}\left(x_{k}\right)$ for $k \in \mathcal{J}$; thus, as seen in the previous section, the last two terms in equation (8) cancel each other.

This formulation can be useful in two situations: enrichment and coupling of finite elements and mesh-free approximation. In the first one a complete finite element base is considered all over the domain, and only in a selected region, particles are added increasing the consistency of the approximation, see [3] for details.

However, in this paper only coupling with finite elements is considered. That is, as it is shown in Figure 4, the domain $\Omega$ is the union of two non-disjoint subdomains, $\Omega=\Omega^{h} \cup \Omega^{\rho}$. Here $\Omega^{h}$ denotes the subdomain where the $N_{j}^{h}$ have an influence in the approximation,

$$
\Omega^{h}=\left\{x \in \Omega \mid \exists j \in \mathcal{J}, N_{j}^{h}(x) \neq 0\right\},
$$

and $\Omega^{\rho}$ is the subdomain where at least one $N_{j}^{\rho}$ is non zero,

$$
\Omega^{\rho}=\left\{x \in \Omega \mid \exists i \in \mathcal{I}, N_{i}^{\rho}(x) \neq 0\right\}
$$




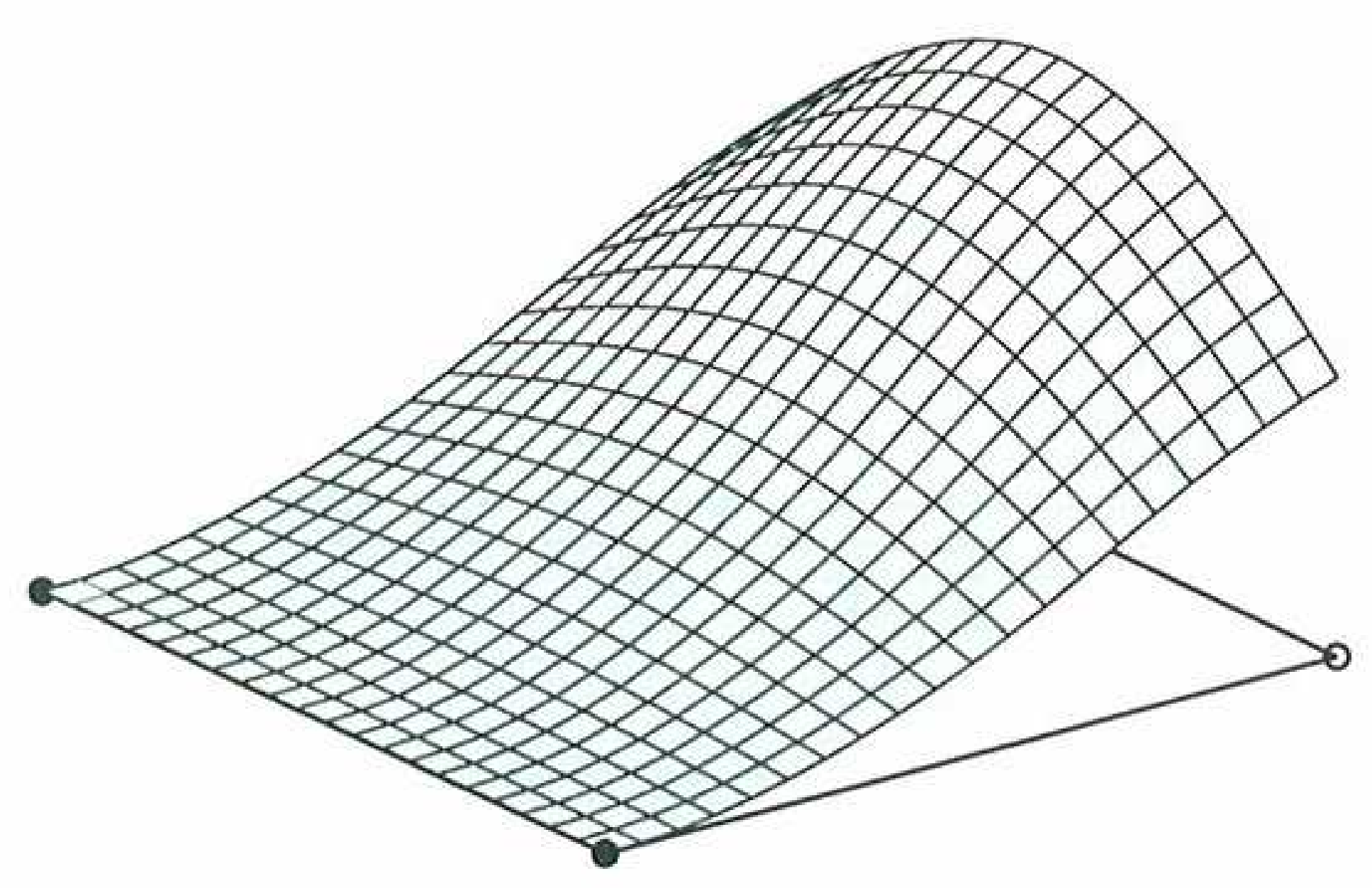

Fig. 5. Continuous blending mesh-free shape function over an element with two active nodes.

In the region where only finite elements are present, $\Omega^{h} \backslash \Omega^{\rho}$, a standard, and thus consistent, finite element approximation is considered. In the region where only particles have an influence, $\Omega^{\rho} \backslash \Omega^{h}$, the standard, and thus consistent, EFG approximation is considered. However, in the area where both interpolations have an influence, $\widetilde{\Omega}:=\Omega^{h} \cap \Omega^{\rho}$, the coupled interpolation is used, see the definition of $\widetilde{u}(x)$ in (4).

It is important to note that continuity of the interpolation is ensured by the following proposition under the described conditions even in multiple dimensions, see [4] for a demonstration.

Proposition 1 The approximation $\widetilde{u}(x)$ is continuous in $\Omega$ if:

(1) the same order of consistency $m$ is imposed all over $\Omega$ (i.e. $m$ coincides with the degree of the FE base), and

(2) the domain of influence of particles, $\Omega^{\rho}$, coincides exactly with the region where finite elements do not have a complete basis.

If the consistency of the mesh-free interpolation, $m$, coincides with the degree of the base of finite elements and the base is complete, then $\mathbf{P}(x)-\pi^{h} \mathbf{P}(x)=$ 0 , and thus, from (7), $\widetilde{\alpha}=0$. That is, if a uniform consistency is required all over the domain $\Omega$, in the regions where the finite element base is complete, i.e. $\Omega \backslash \Omega^{\rho}$, the contribution of the particles is zero. Note that this property holds at every point $x \in \Omega \backslash \Omega^{\rho}$ and, in particular, along the boundaries of the finite element mesh. As shown in Figure 5, this means that $\widetilde{N}_{i}^{\rho}=0$ in the finite element edges (or faces in 3D) whose nodes are all in $\mathcal{I}$. This is an important property for the imposition of essential boundary conditions, as seen in section 3.2 .

Obviously in the rest of the domain, $\Omega^{\rho}$, the mesh-free shape functions take care of reproducing polynomials up to degree $m$.

Remark 6 From the definition of the approximation $\widetilde{u}(x)$ in (4) it is easy to 
verify that now the approximation space is

$$
\widetilde{\mathcal{S}}:=\operatorname{span}\left\{N_{j}^{h}, j \in \mathcal{J}\right\} \oplus \operatorname{span}\left\{\widetilde{N}_{i}^{\rho}, i \in \mathcal{I}\right\} .
$$

Note that the shape functions $\left\{N_{j}^{h}\right\}_{j \in \mathcal{B}}$ and $\left\{\widetilde{N}_{i}^{\rho}\right\}_{i \in \mathcal{I}}$ are linearly independent. That is, the only possible combination of parameters $\alpha_{i}, i \in \mathcal{I}$, and $\beta_{j}, j \in \mathcal{J}$, such that

$$
\sum_{j \in \mathcal{J}} \alpha_{j} N_{j}^{h}+\sum_{i \in \mathcal{I}} \beta_{i} \widetilde{N}_{i}^{\rho}=0
$$

is the trivial one. Note that, on one hand, in $\Omega \backslash \Omega^{\rho}$ every $\widetilde{N}_{i}^{\rho}$ is zero and the finite element shape functions are linearly independents, i.e. the only possible combination of parameters $\alpha_{j}$ such that $\left.\sum_{j \in \mathcal{J}} \alpha_{j} N_{j}^{h}\right|_{\Omega \backslash \Omega^{\rho}}=0$ is the trivial one. And, on the other hand, in $\Omega^{\rho}$ the moving least square procedure to determine $\widetilde{N}_{i}^{\rho}$ from (5) and (7) ensures linear independency.

\section{Comparing both formulations}

\subsection{General remarks}

As seen in Remarks 2 and 6 the interpolation spaces corresponding to the bridging scale method and the continuous blending method are not equivalent

$$
\begin{aligned}
\widehat{\mathcal{S}}=\operatorname{span}\left\{N_{j}^{h}, j \in \mathcal{B}\right\} \oplus \operatorname{span} & \left\{N_{i}^{\rho}, i \in \mathcal{I}\right\} \\
& \neq \widetilde{\mathcal{S}}=\operatorname{span}\left\{N_{j}^{h}, j \in \mathcal{B}\right\} \oplus \operatorname{span}\left\{\widetilde{N}_{i}^{\rho}, i \in \mathcal{J}\right\} .
\end{aligned}
$$

In fact, equations (1) and (8) also illustrate the differences. The last term in the r.h.s. of both equations is clearly different; note that the constant value $N_{i}^{\rho}\left(x_{j}\right)$, which appears in (1), is replaced by the function $\overline{\bar{N}}_{i}^{\rho}\left(x, x_{j}\right)$ in (8). As noticed in Remark 5 , there is a coincidence at $x=x_{j} j \in \mathcal{B}$ because both approaches are hierarchical, but elsewhere the differences have important consequences:

(1) Lower computational cost of continuous blending method. Both formulations require the determination of mesh-free shape functions. Thus at each integration point a system of equations must be solved. In the continuous blending method the system is defined in (7), for the bridging scale method the same system is solved but without the second term on the r.h.s. Apart from this, on one hand, the bridging scale method requires the computation of the projection of the standard mesh-free shape functions onto de finite element space, $\pi^{h} N_{i}^{\rho}$. That is, the standard mesh-free shape functions, $N_{i}^{\rho}$, must be computed at every active finite element node, $\left\{x_{j}\right\}_{j \in \mathcal{B}}$. On the other hand, the continuous blending coupled interpolation requires only the computation of the projection of the polynomial base $\mathbf{P}$ at some finite element nodes. That is, it requires 
the computation of $\mathbf{P}\left(x_{j}\right)$ only at the nodes $x_{j}$ in the transition region $\widetilde{\Omega}$, which is easily implemented.

(2) The continuous blending method requires particles only where needed. As observed in Section 2.2 in the continuous blending method the mesh-free contribution is cancelled in the region where the finite element base is complete. Thus, there is no need to have any particles in this region. This is not the case for the bridging scale method. For this approach a complete mesh-free approximation is needed in the whole domain in order to ensure the continuity of the approximation. Obviously, this increases the total number of degrees of freedom. If finite elements are only used along the Dirichlet boundary to introduce essential boundary conditions, the number of degrees of freedom may not increase because the region where the finite element base is complete is reduced to the Dirichlet boundary. But in this last case the next comparison is crucial.

(3) Dirichlet boundary conditions are properly imposed by finite elements only in the continuous blending method. As noted in Section 2.1, and in particular in Figure 2, the bridging scale mesh-free shape functions, $\widehat{N}_{i}^{\rho}$ do not vanish at the element edges (or faces in 3D) where a complete finite element base is present; for instance, along the essential boundary. Thus, prescribing the essential boundary at the active finite element nodes, i.e. imposing the coefficients $a_{j}$ in (2), does not imply that the Dirichlet condition is "exactly" (in the sense of the consistency required) imposed along this boundary. Moreover, and in fact more importantly, the test functions do not cancel along the Dirichlet boundary decreasing the optimal rate of convergence. As noted in $[8,10]$ this fact forces the use of a modified weak form in order to impose the essential boundary condition in a correct manner. Note that this problem is not present in the continuous blending method because the mesh-free shape functions cancel when the finite element base is complete, see Figure 5.

To better illustrate this last issue a particular example is presented. A square domain is discretized by means of finite element nodes and particles. A regular net of particles is used all over the square domain, and active nodes are added along the boundary as shown in Figure 6 (top pictures). Thus, nodes and particles coincide along the boundary.

Figure 6 also shows the shape function associated to a mesh-free particle (depicted in gray) for both formulations. On the left, the particle is chosen along the boundary. This represents the limit case when particles get close to the boundary, because the same behavior is also present when the particle is not located exactly on the boundary as shown on the right of the same figure. The results are clear, for the bridging scale method (middle picture) the shape function clearly does not cancel along the boundary. Thus as noted previously, imposing the Dirichlet conditions only at the finite element nodes does not suffice. For the continuous blending method, this is not the case, the shape 

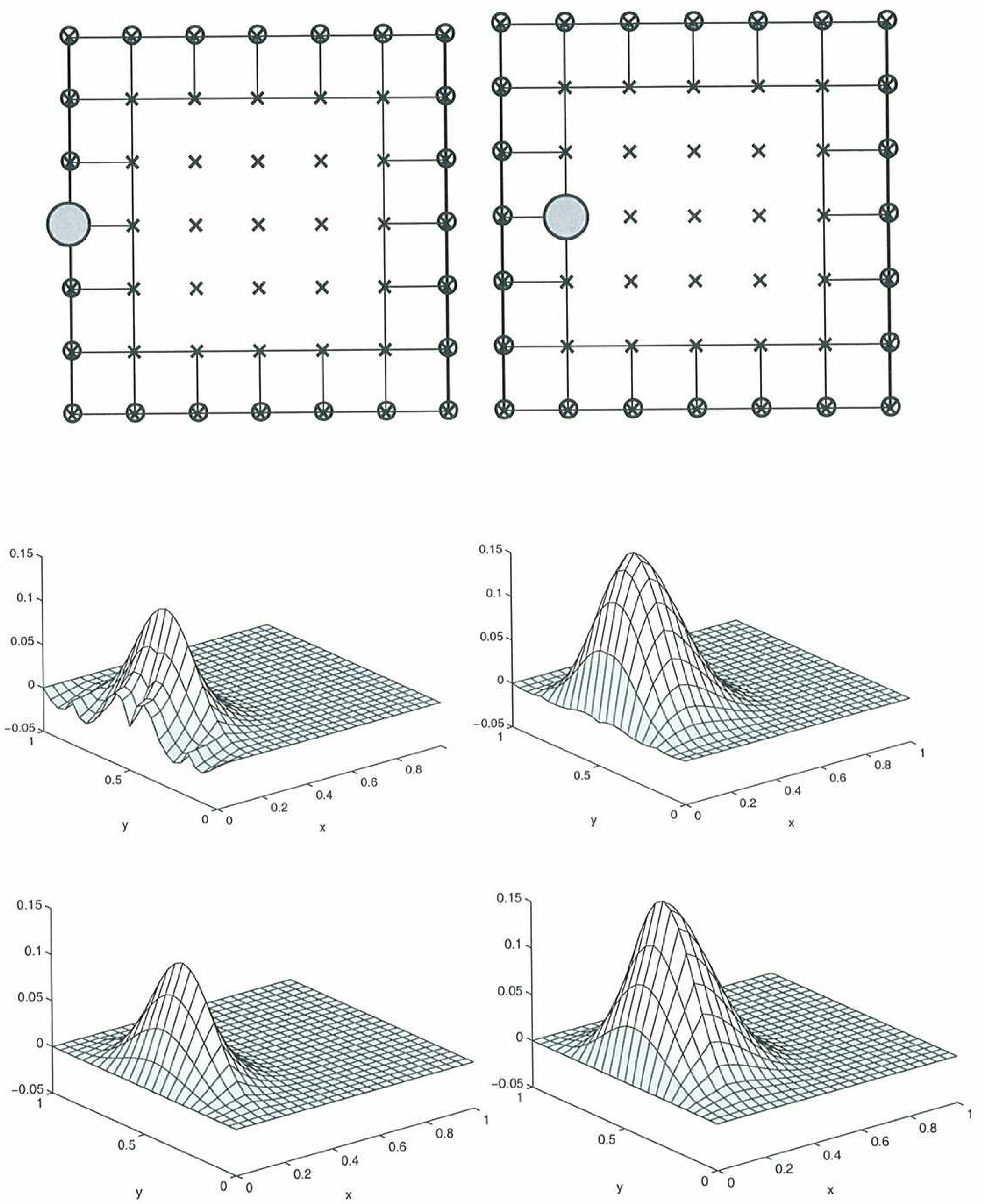

Fig. 6. Discretization with finite element nodes (o) and particles (x) (top), and shape function associated to the particle located at the gray circle for the bridging scale method (middle) and for the continuous blending method (bottom).

function cancels along the boundary. On the right, the particle is chosen one layer inside the domain, and although less evident, the same conclusions are drawn. 


\subsection{Numerical example}

The same example presented in [8] is repeated here to compare both approaches. The 2-D Laplace equation is solved on the unit square with Dirichlet boundary conditions,

$$
\begin{array}{ll}
\Delta u=0 & \text { for }(x, y) \in \Omega:=] 0,1[\times] 0,1[, \\
u(x, 1)=0 \text { and } u(x, 0)=\sin (\pi x) & \text { for } x \in] 0,1[ \\
u(0, y)=u(1, y)=0 & \text { for } y \in] 0,1[.
\end{array}
$$

The analytical solution is simply,

$$
u(x, y)=(\cosh (\pi y)-\operatorname{coth}(\pi y) \sinh (\pi y)) \sin (\pi x) .
$$

The Galerkin weak problem associated to (9) becomes: find $u \in \mathcal{S}$ such that, for all $v \in \mathcal{V}$,

$$
\int_{\Omega} \nabla u \cdot \nabla v d \Omega=0
$$

where the functional spaces are, as usual, the solution space,

$$
\mathcal{S}:=\left\{u \in \mathcal{H}^{1}(\Omega) \mid u \text { verifies (9b) and (9c) }\right\} \text {, }
$$

and the test space, $\mathcal{V}:=\mathcal{H}_{0}^{1}(\Omega)$, such that the test functions $v$ vanish at the essential boundary (recall, this is not exactly verified by the bridging scale method). The finite dimensional subspaces have similar properties, the test functions vanish along the Dirichlet boundary and the approximations verify (up to the interpolation error) the essential boundary conditions. Recall that these conditions are easily imposed in the continuous blending method by prescribing only the values at the finite element nodes. Thus the standard theoretical convergence rates are easily obtained with this formulation. However, with the bridging scale method this is not the case, and a reduction in the convergence rates is expected as shown in [9].

Figure 7 shows the results obtained with the continuous blending method and the discretization shown in the same figure. Finite elements are considered in a neighborhood of the boundary and only the nodes at the boundary are used in the interpolation (marked with circles). The finite element shape functions are coupled with the mesh-free shape functions (see the particles marked with crosses) as commented in section 2.2. The solution is correctly interpolated at the boundaries and vanishes at $\{x=0\},\{x=1\}$ and $\{y=1\}$. The reason being that a complete finite element base is available at the boundary and, therefore, the interpolation is made exclusively with finite element shape functions. Thus, it is piecewise linear. The interpolation of the essential boundary condition is easily improved with the discretization shown in Figure 8, where more finite element nodes are used at $\{y=1\}$. 

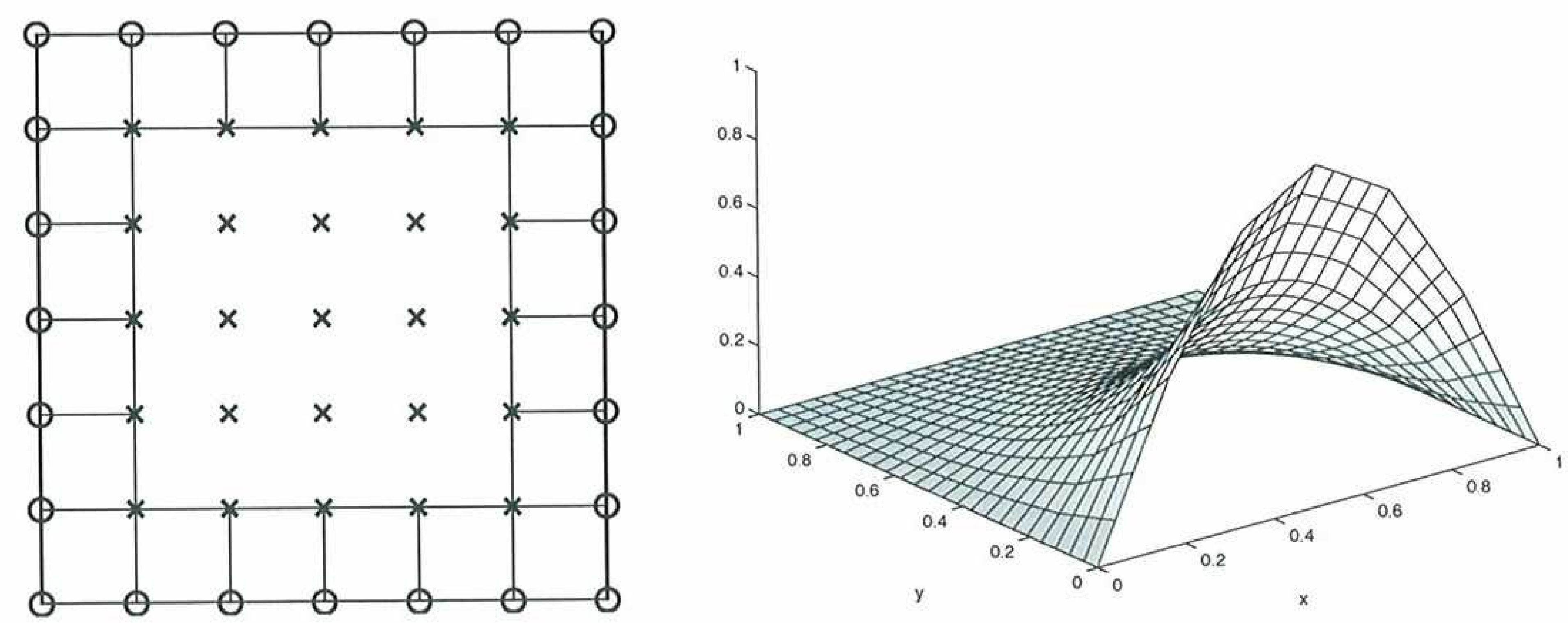

Fig. 7. Continuous blending method: discretization and interpolation.
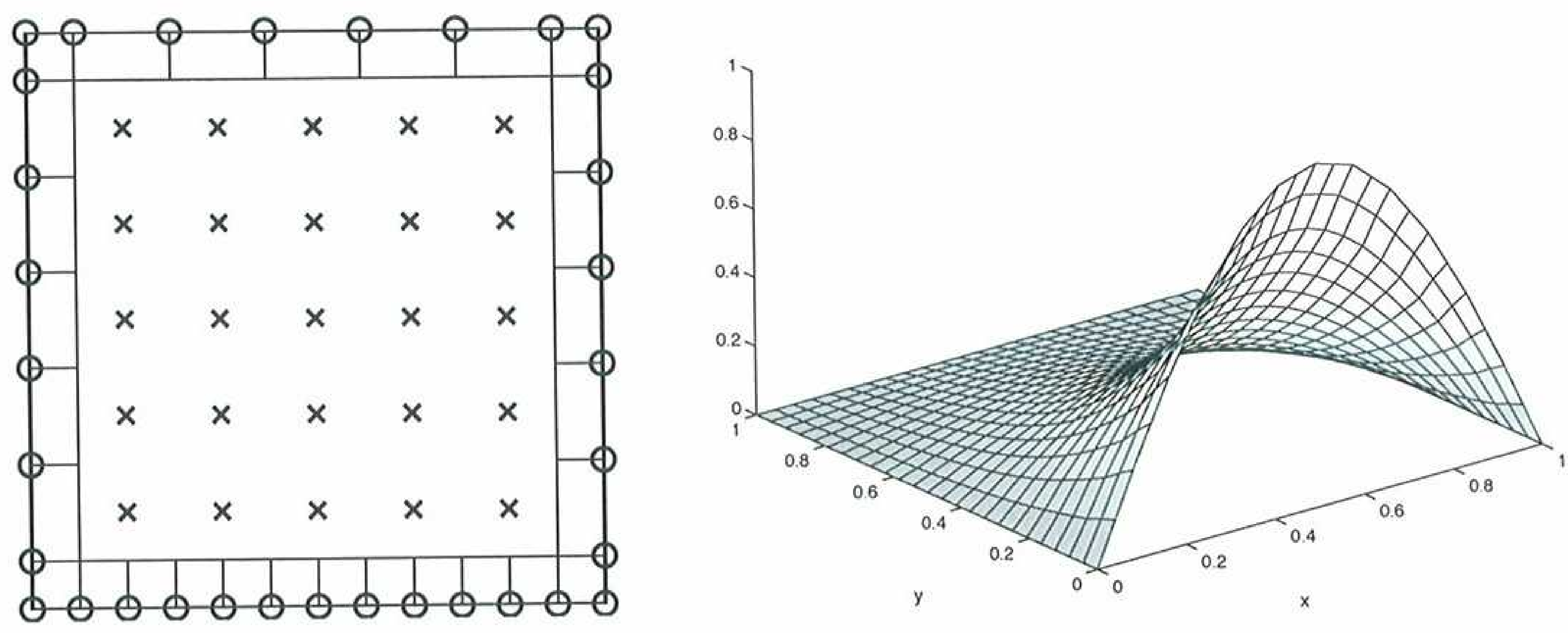

Fig. 8. Continuous blending method: refined finite element discretization along the boundary.

Figure 9 shows the solution obtained with the bridging scale method presented in section 2.1. The numerical solution presents an important error at the essential boundary, even when a finer mesh is used, see Figure 10. Although the mesh-free shape functions vanish at the finite element nodes, they do not vanish along the essential boundary.

Finally, convergence results are presented in Figure 11 where the rates of both methods and standard finite elements are compared. On the left convergence in the $\mathcal{L}_{2}(\Omega)$ norm is presented. It is difficult to appreciate from this figure the difference in convergence rates between both techniques blending finite elements and mesh-free methods because the error is measured over the whole computational domain. Thus, on the right, convergence is plotted using the $\mathcal{L}_{2}(\partial \Omega)$ norm. In this case it is clear that both finite elements and the continuous blending method technique presents identical results and the optimal rate of convergence. This is not the case for the hierarchical coupling, which, 

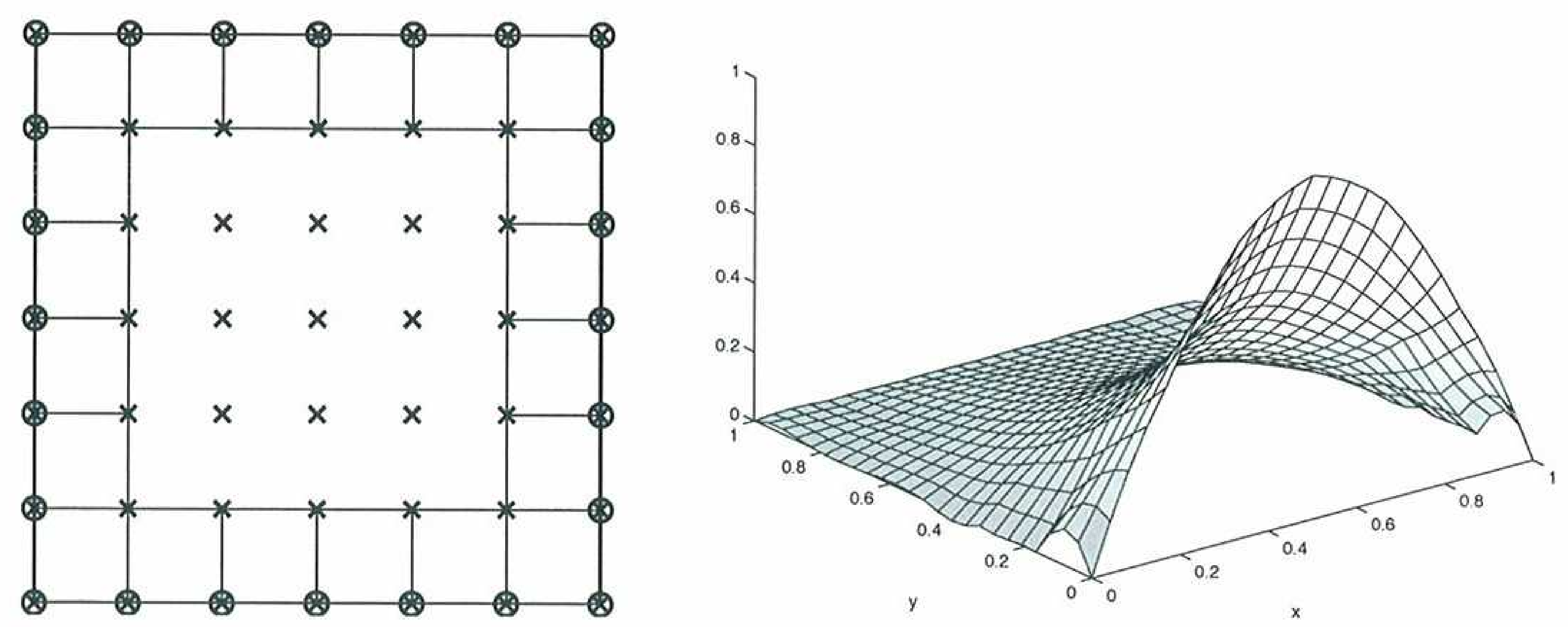

Fig. 9. Bridging scale method: discretization and interpolation.
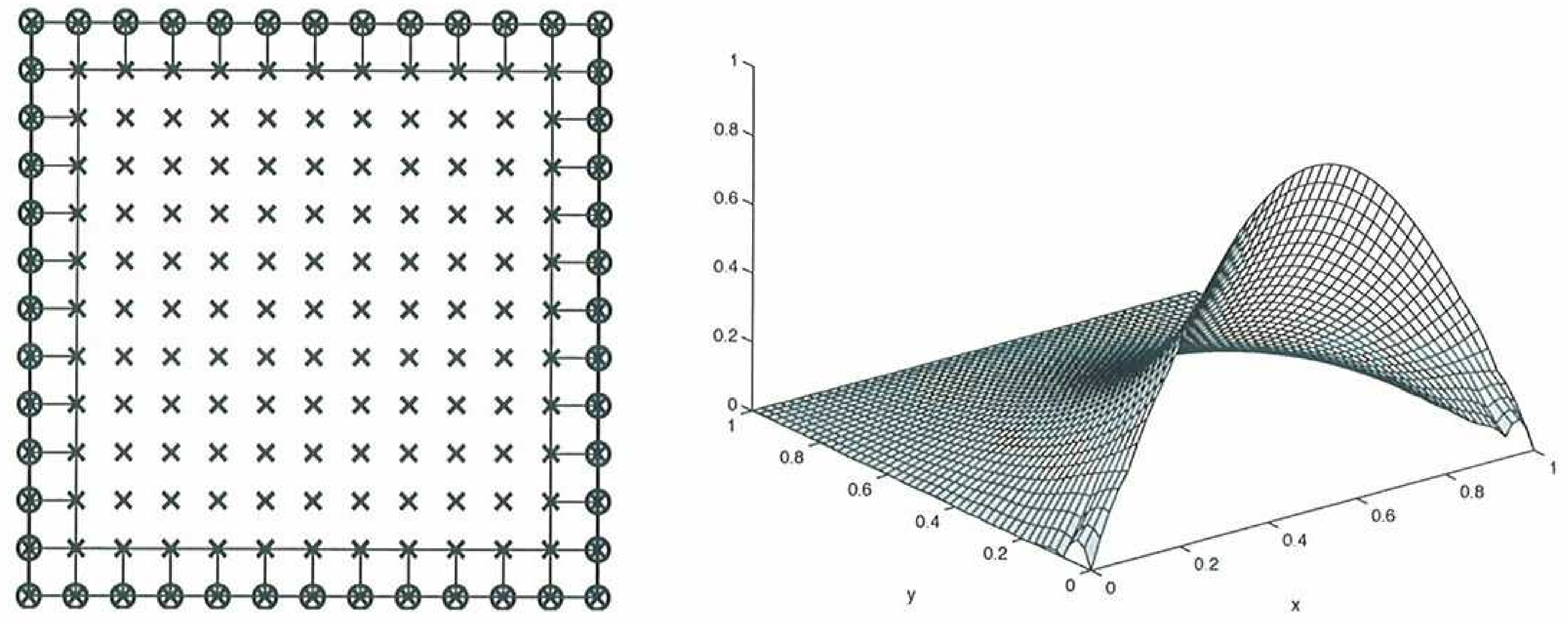

Fig. 10. Bridging scale method: refined discretization.

as expected, shows a reduced convergence rate.

\section{Conclusions}

The bridging scale method proposed in $[7,8]$ is compared with the continuous blending method proposed in [3]. Both formulations are hierarchical and preserve the desired consistency on the computational domain. Thus, for a large number of situations they are equivalent. However, apart from computational efficiency (the continuous blending method presents advantages from a computational point of view) the major difference is apparent when finite elements are employed to impose the essential boundary conditions. The continuous blending method is such that the mesh-free shape functions cancel when a complete finite element base is present. This also happens along boundaries 

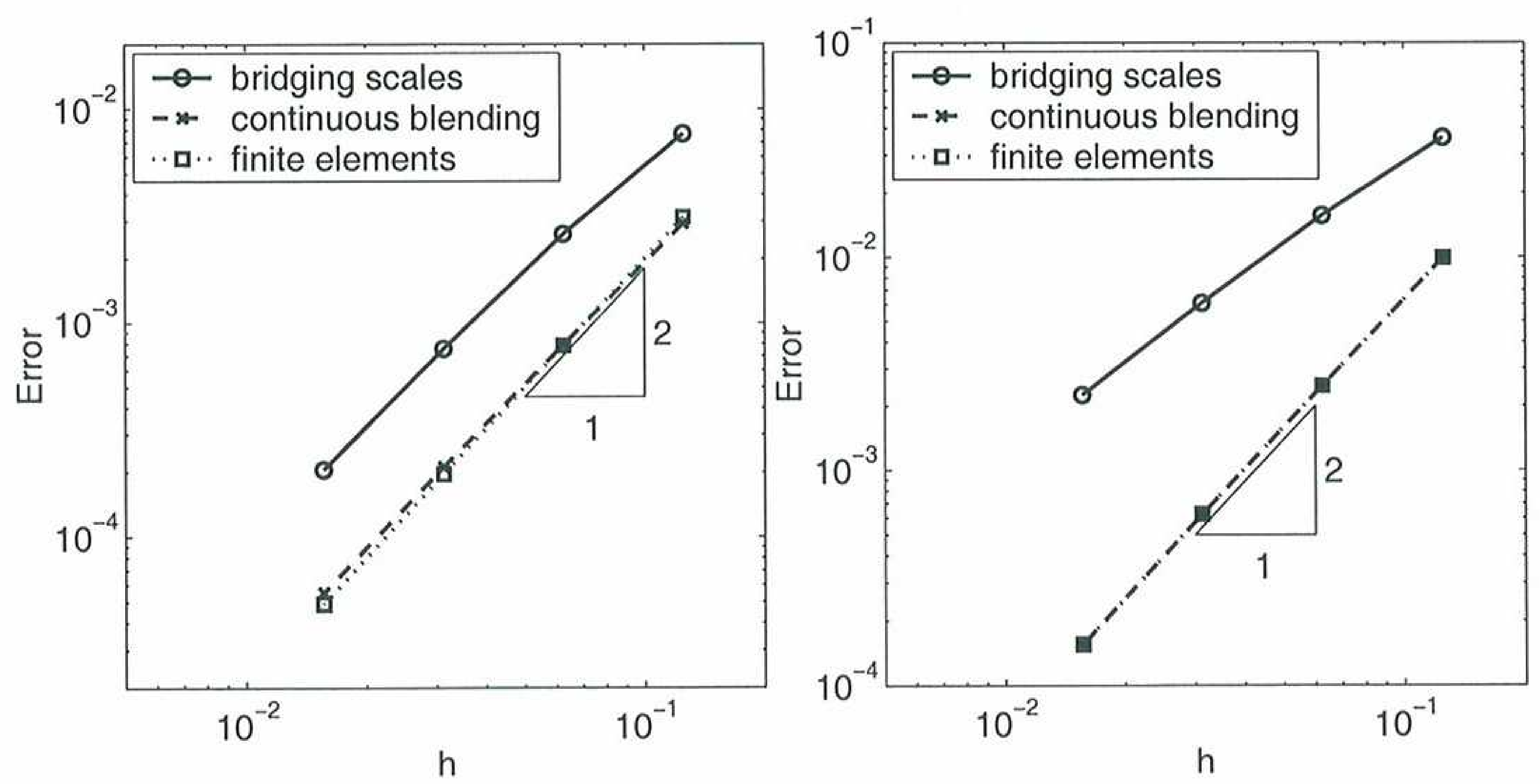

Fig. 11. Convergence comparison with $\mathcal{L}_{2}(\Omega)$ (left) and $\mathcal{L}_{2}(\partial \Omega)$ (right).

(edges or faces of the finite element mesh). This property, which allows to put mesh-free methods only where they are needed, is crucial to impose in a simple manner essential boundary conditions and, more importantly, to ensure that the test functions cancel along the Dirichlet boundary. In fact, the continuous blending method preserves the optimal order of convergence of mesh-free and finite elements. This is not the case of the bridging scale method.

\section{References}

[1] T. Belytschko, D. Organ, Y. Krongauz, A coupled finite element-element-free Galerkin method, Comput. Mech. 17 (3) (1996) 186-195.

[2] D. Hegen, Element free galerkin methods in combination with finite element approaches, Comput. Methods Appl. Mech. Eng. 135 (1996) 143-166.

[3] A. Huerta, S. Fernández-Méndez, Enrichment and coupling of the finite element and meshless methods, Int. J. Numer. Methods Eng. 48 (11) (2000) 1615-1636.

[4] S. Fernández-Méndez, A. Huerta, Coupling finite elements and particles for adaptivity: An application to consistently stabilized convection-diffusion, in: M. Griebel, M. A. Schweitzer (Eds.), Meshfree methods for partial differential equations, Vol. 26 of Lecture Notes in Computational Science and Engineering, Springer-Verlag, Berlin, 2002, pp. 117-129, papers from the International workshop, Universität Bonn, Germany, September 11-14, 2001.

[5] A. Huerta, S. Fernández-Méndez, P. Díez, Enrichissement des interpolations d' éléments finis en utilisant des méthodes de particules, ESAIM-Math. Model. Numer. Anal. 36 (6) (2002) 1027-1042.

[6] S. Fernández-Méndez, P. Díez, A. Huerta, Convergence of finite elements enriched with meshless methods, Numer. Math. To appear. 
[7] W. K. Liu, R. A. Uras, Y. Chen, Enrichment of the finite element method with the reproducing kernel particle method, J. Appl. Mech., ASME 64 (1997) 861-870.

[8] G. J. Wagner, W. K. Liu, Hierarchical enrichment for bridging scales and meshfree boundary conditions, Int. J. Numer. Methods Eng. 50 (3) (2001) 507-524.

[9] W. Han, G. J. Wagner, W. K. Liu, Convergence analysis of a hierarchical enrichment of Dirichlet boundary conditions in a mesh-free method, Int. J. Numer. Methods Eng. 53 (6) (2002) 1323-1336.

[10] L. Zhang, G. Wagner, W. K. Liu, A parallelized meshfree method with boundary enrichment for large-scale CFD, J. Comput. Phys. 176 (2) (2002) 483-506.

[11] W. K. Liu, S. Jun, Y. F. Zhang, Reproducing kernel particle methods, Int. J. Numer. Methods Fluids 20 (8-9) (1995) 1081-1106.

[12] S. Li, W. K. Liu, Meshfree and particle methods and their applications, Appl. Mech. Rev. 55 (4) (2002) 1-34.

[13] W. K. Liu, S. Li, T. Belytschko, Moving least square reproducing kernel methods Part I: Methodology and convergence, Comput. Methods Appl. Mech. Eng. 143 (1-2) (1997) 113-154.

[14] J.-S. Chen, W. Han, Y. You, X. Meng, A reproducing kernel method with nodal interpolation property, Int. J. Numer. Methods Eng. 56 (7) (2003) 935-960.

[15] A. Huerta, S. Fernández-Méndez, Coupling element free Galerkin and finite element methods, in: Proceedings of the European Congress on Computational Methods in Applied Sciences and Engineering (ECCOMAS 2000), 11-14 September, Barcelona, 2000, electronic publication ISBN: 84-89925-70-4. 